\title{
DESIGN AND IMPLEMENTATION OF A DENSITY-BASED TRAFFIC LIGHT CONTROL WITH SURVEILLANCE SYSTEM
}

\author{
Y. N. Udoakah ${ }^{1, *}$ and I. G. Okure ${ }^{2}$ \\ 1,2 Dept. of Electrical/Electronics \& Computer EnGR', University of Uyo, Uyo, AKWa Ibom STATE. NiGERIA \\ E-mail addresses: ${ }^{1}$ yeobongudokah@uniuyo.edu.ng, ${ }^{2}$ okure74@gmail.com
}

\begin{abstract}
Traffic congestion especially at road intersections is becoming an issue for which road traffic users contend with daily. The conventional traffic light applies a fixed logic of allocating equal " $\mathrm{GO}$ " time to lanes of traffic at road intersections irrespective of the density of traffic on each lane. Using the PIC18F4550 microcontroller interfaced with infrared sensors, a new traffic light control system was developed to ease the flow of traffic at a particular troubled spot in Uyo Metropolis (Abak Road, Udo Eduok, Udo Obio streets intersection) in Akwa Ibom State, Nigeria. Simulation of the proposed design was performed using the Proteus Software while implementation was carried out on a prototype. Performance evaluation of the prototype implemented showed that the 4 arrays (IR Transmitter and Receiver) of infrared sensors interfaced with the microcontroller using AND gates and strategically positioned to read the density of traffic on each lane at the intersection triggered when a vehicle comes between the transmitter and the receiver. Obtaining inputs from these sensors together with the logic from the microcontroller, a new traffic light control system was developed capable of controlling traffic based on the density of each lane of traffic. Results obtained from simulation and implementation of the design indicates that the traffic control system with the PIC18F4550 microcontroller and the infrared sensors gives a better performance compared to the conventional traffic light control system.
\end{abstract}

Keywords: Road traffic density, Traffic light, Uyo metropolis, '+' road intersection, Proteus.

\section{INTRODUCTION}

The constant movement of people from rural to urban areas in search of greener pastures has resulted in urban population explosion and over-stretched infrastructures. One of such over-stretched infrastructure is the road, a situation which has culminated to increased traffic. Although traffic lights have always been used for controlling the movement of traffic (pedestrian or automobile), traffic management in major cities around the world has continued to be a subject of concern. In an attempt to tackle the traffic management concern, various designs have either been proposed or implemented. The first of such installation dates back to 1868 [1-2]. Since then, modern day civilization has found application in its use for managing traffic in cities in the absence of traffic wardens or officers; though with innovations, changes have been made in signs, but the working principle for traffic and pedestrian control remains the same.

With the rapid growth in the population of our cities, the busy nature of our roads and the need to maximize one's time, there arise a need for a more dynamic, systematic and efficient traffic management schemeone which seeks to reduce unnecessary congestion and lawlessness resulting from undue delays. It would be imperative that a new design be developed so as to enhance the flow and control of traffic using the density of vehicles on a particular lane by automatically assigning the left-over time in an idle lane to the lane with more traffic density, hence, reducing traffic congestion in those lanes and at the same time capture the vehicle license number plate of road users who disobey traffic using the surveillance system. The aim of this work is therefore to design a density based traffic light control system with a surveillance monitoring system using the technology which is similar to that of [1].

Studies have been carried out all with the aim of improving the traffic management system; [3] described the modeling and implementation of an advanced traffic signal controller within a microscopic simulation environment, MITSIMLab. The controller was designed with a generic and flexible logic that allowed it to simulate control strategies including both

* Corresponding author, tel: +234-806-688-7235 
isolated and coordinated intersection control, with fixed-time and demand responsive logic; implementation was carried out through a case study in which it was applied to an urban arterial network in Stockholm, Sweden. Similarly, [4] proposed the optimization of the traffic light controller in a city using ultrasonic sensor and microcontroller with fault detection technique. Their proposal included an idea for a dynamic and automatic traffic light control expert system combined with a simulation mode. [5] designed a traffic system that was capable of receiving signal from emergency vehicles based on radio frequency (RF) transmission and used the Programmable Integrated Circuit (PIC) 16F877A microcontroller to change the sequence back to the normal sequence before the emergency mode was triggered. [6] designed and implemented a traffic light system for road intersection control; using image processing technique, [7] proposed the design of an intelligent traffic control system and noted that by continuously sensing and adjusting the timing of traffic lights according to the actual traffic load, the traffic congestion problem could be solved. [8] employed a method proposed to reduce heavy traffic congestion on the road by using PLC based traffic diversion system; employing the working of weight sensors whose output were fed to a PLC to control the traffic diversion. It could be seen from various studies that traffic system management is critical and in view of this, there is need for the design of a simple and cost effective traffic management control system devoid of complexities but capable of tackling most of the traffic concerns raised. This is what this work seeks to achieve.

The major components used in implementing this design are: the 5V DC power supply unit, infrared sensors array and the PIC18F4550 microcontroller. The PIC18F4550 a 40-pin PDIP with 20 pins on each row and 256 bytes of EEPROM (Electrically Erasable and Programmable Read Only Memory), 2KB of SRAM (Static RAM) and $32 \mathrm{~KB}$ of flash memory.

\section{METHODOLOGY AND SYSTEM DESIGN}

\subsection{Design for Density Based Traffic Light Control System}

The approach to this design is realized through the design and implementation of its input subsystem, control unit (control program) and output subsystem. The input subsystem is made of sensors, programmed and implemented using some already existing principles to achieve optimum performance. The control unit is realized by a microcontroller-based control program, which interprets the input and qualifies it to produce a desired output. The block diagram of the entire system as presented in Figure 1 shows the major components of the system.

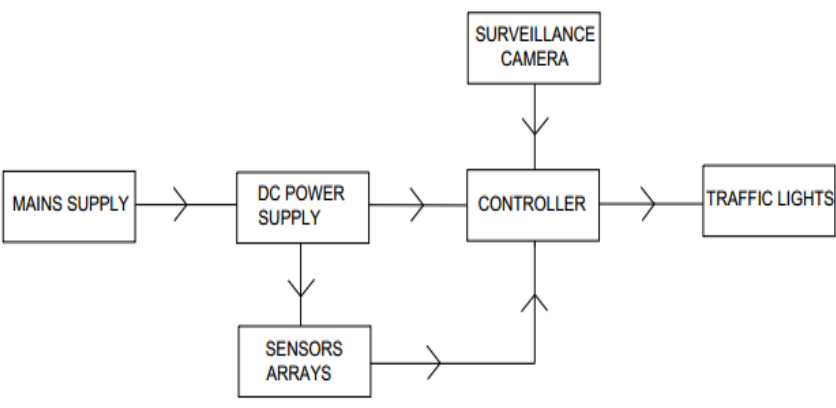

Figure 1: Block diagram of the Density Based Traffic Light Control System.

These include:

- Mains Supply

- DC Power Supply

- $\quad$ Sensors Arrays

- Controller

- $\quad$ Traffic Lights

- $\quad$ Surveillance Camera

The block diagram was drawn so as to give a vivid explanation of how the system works at a glance, thus; the main supply provides 230VAC power which is converted to 5VDC (VDD) by the DC power supply used to power the sensor arrays, the controller, the surveillance camera and traffic lights. The sensors provide input to the controller which then performs some logical operations to power the traffic lights as output used for controlling traffic at road intersections. Additionally for the proposed surveillance system, the camera is interfaced with the controller to capture license number plates of traffic defaulters for storage and law enforcement purposes.

\subsubsection{Choice of Sensors for Sensors Arrays}

In choosing the sensors, the following features were taken into consideration: accuracy, range, calibration, resolution and affordability. Although the infrared (IR) sensors are usually disturbed by noise in the surrounding such as radiations, ambient light etc., they were used for this design because they are cheap and readily available in the market and are easy to interface.

\subsubsection{Choice of Microcontroller and Peripherals}

Although the microcontroller PIC18F4550 has a few disadvantages relating to its possession of a single accumulator, but it was chosen because of the following features noted in [9]:

- Most effective and efficient microcontroller to be used to accomplish the objective of the design.

- $\quad$ Low cost, low percentage of faulty rate and very consistent.

Vol. 36, No. 4, October 2017 
- $\quad$ More pins available for use in implementation of the design features.

- Very fast because of its RISC architecture.

- Low power consumption and ease of programming.

- $\quad$ Ease of interfacing of an analog device without any extra circuitry.

The pin description of the microcontroller is shown in Table 1 while Figure 2 shows the interfacing of the microcontroller with the peripherals. The 40 pins of PIC18F4550 shown in Figure 3 are divided into 5 ports. Out of which, 35 pins are Input-Output pins which can be configured for general input or output by setting registers associated with them.

Table 1: Ports and pin-outs on PIC18F4550.

\begin{tabular}{ccc}
\hline Ports & Number of Pins & Pin Names \\
\hline PORT A & 7 & RA0-RA6 \\
PORT B & 8 & RB0-RB7 \\
PORT C & 7 & RC0-RC2, RC4-RC7 \\
PORT D & 7 & RD0-RD7 \\
PORT E & 4 & RE0-RE3 \\
\hline
\end{tabular}

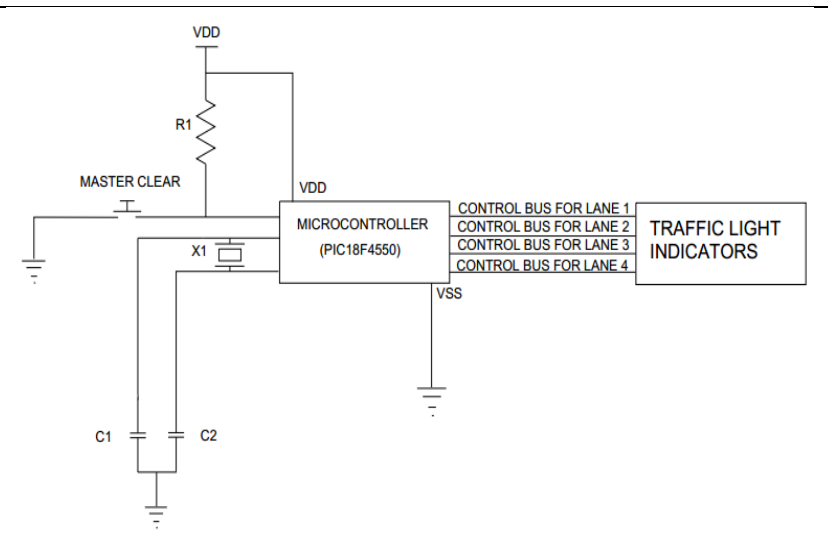

Figure 2: Interfacing PIC18F4550.

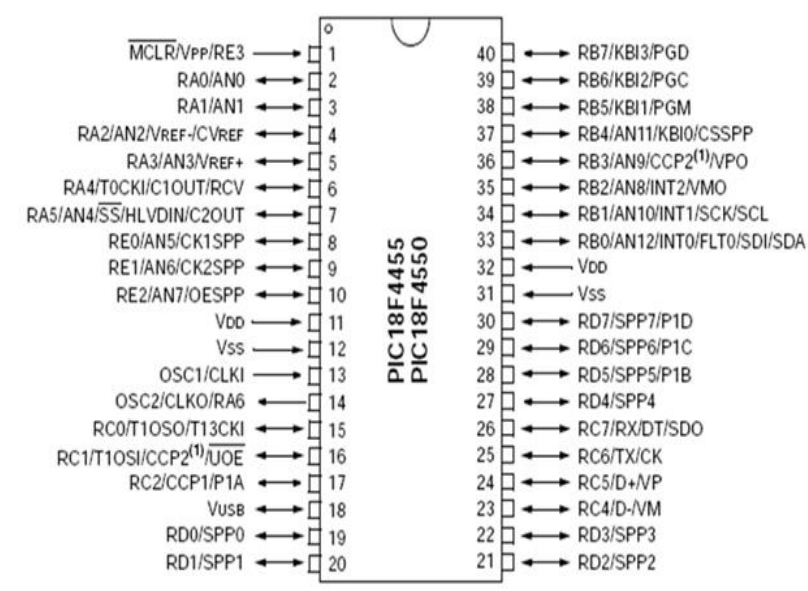

Figure 3: Pin layout of PIC18F4550 [10].

Each port in the microcontroller is associated with three 8 bit registers [10] for IO operations.
- $\quad$ TRISX (8 bit): where $X$ represents the name of the ports. (A, B, C, D, E, etc). This register assigns the direction of the pins (Input or Output). For example "TRISB $=0 x F 0$ ", will set all the pins in port B to Output.

- $\quad$ LATX (8 bit): The latch registers reads and modifies the write operation on the value of $\mathrm{I} / 0$ pin and stores the output data that is to be passed on to the external hardware.

- $\quad$ PORTX (8 bit): Reads the device level, stores the input level of the pins and registers the input signal from the external device if the pin is configured as input.

\subsubsection{Choice of the Crystal Oscillator}

The crystal oscillator $X 1$ in Figure 2 used in this design for the interfacing of the microcontroller is of the frequency $4 \mathrm{MHz}$ as recommended by the manufacturer of the microcontroller.

From the $R-L-C$ values given by [11], a $4 \mathrm{MHz}$ crystal oscillator should have the following $R-L-C$ values;

$R=100 \Omega, \quad L=100 \mathrm{mH}, \quad C=0.015 p F$

The series-resonant frequency $f_{s}$ when the inductive reactance $X_{L}$ is equal to the capacitive reactance $X_{C}$ can be calculated using Eq. (1) below;

$$
\begin{gathered}
f_{s}=\frac{1}{2 \pi \sqrt{L C}}=\frac{1}{2 \pi \sqrt{100 \times 10^{-3} \times 0.015 \times 10^{-12}}} \\
=4.10 \mathrm{MHz}
\end{gathered}
$$

\subsubsection{Choice of Ceramic Capacitor}

The value of capacitors $\mathrm{C} 1$ and $\mathrm{C} 2$ in Figure 2 is $22 \mathrm{pF}$ which falls within the range of $20 p F-40 p F$ recommended by the microcontroller manufacture. The capacitance of the capacitor can be found using Eq. (2) thus:

$$
\begin{gathered}
C=\varepsilon_{O} \frac{A}{d} \\
\varepsilon_{o}=8.84 \times 10^{-12} \mathrm{~F} / \mathrm{m} ; A=0.015 \mathrm{~m}^{2} ; d=6 \times \\
10^{-3} \mathrm{~m} \\
\quad C=8.84 \times 10^{-12} \times \frac{0.015}{6 \times 10^{-3}}=22.1 p F
\end{gathered}
$$

\subsubsection{Choice of Pull-Up Resistors}

The resistor R1 in Figure 2 is a pull up resistor which is connected between a signal conductor and a positive power supply voltage VDD to ensure that the signal will be a valid logic level. The value of the resistor is determined by the manufacturer of the microcontroller, thus, $10 \mathrm{~K} \Omega$ was used.

\subsubsection{Choice of Traffic Light Indicators}

Just like the conventional traffic light indicator as shown in Figure 4, this design controls traffic using 
three light emitting diodes, 'GREEN', 'YELLOW' and 'RED', each having their usual meaning of 'GO', 'READY' and 'STOP' respectively. They are controlled by the control buses of the microcontroller depending on the logical decisions taken by the controller to control the lanes of traffic according to their densities.

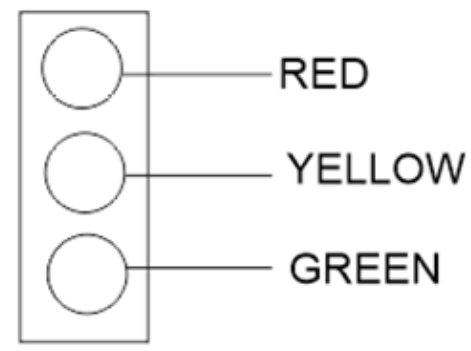

Figure 4: Traffic light indicators.

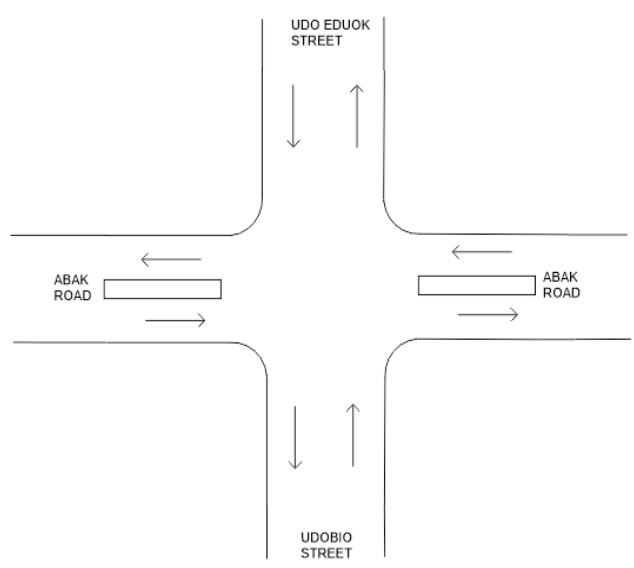

Figure 5: Road layout at point of implementation in Uyo Metropolis.

\section{IMPLEMENTATION}

\subsection{Road Layout}

For the implementation of this design, the Abak road, Udobio and Udo Eduok Street axis in Uyo, Akwa Ibom State, Nigeria was considered. The road layout for this design is the "+" road intersection represented in Figure 5 similar to the road layout used in [12-14].

The trouble spot was chosen and a model developed, because of its constant traffic log-jamb. It was observed that smaller streets like Udo Eduok and Udobio which often times had little traffic were allotted equal 'GO' time when compared to the Abak Road a major expressway.

\subsubsection{Infrared Sensors Arrangement and Implementation}

Since the design is focused on sensing the traffic level on each of the lanes of the road depending on the density of each lane using infrared sensors, the arrangement of the infrared sensor on the road layout was positioned to perform this function.
The sensors are arranged on the median strip or road reservation and on the drainage embankments for Abak Road while using only the drainage embankments or pavements for Udo Eduok and Udobio streets due to the absence of the median strip in these streets. This avoids the problem of probable interference with the infrared rays from the transmitters by pedestrian and stray animals. For each lane of traffic to be controlled, four sensor arrays were deployed and arranged in an array of four, two transmitters and two receivers as shown in Figure 6.

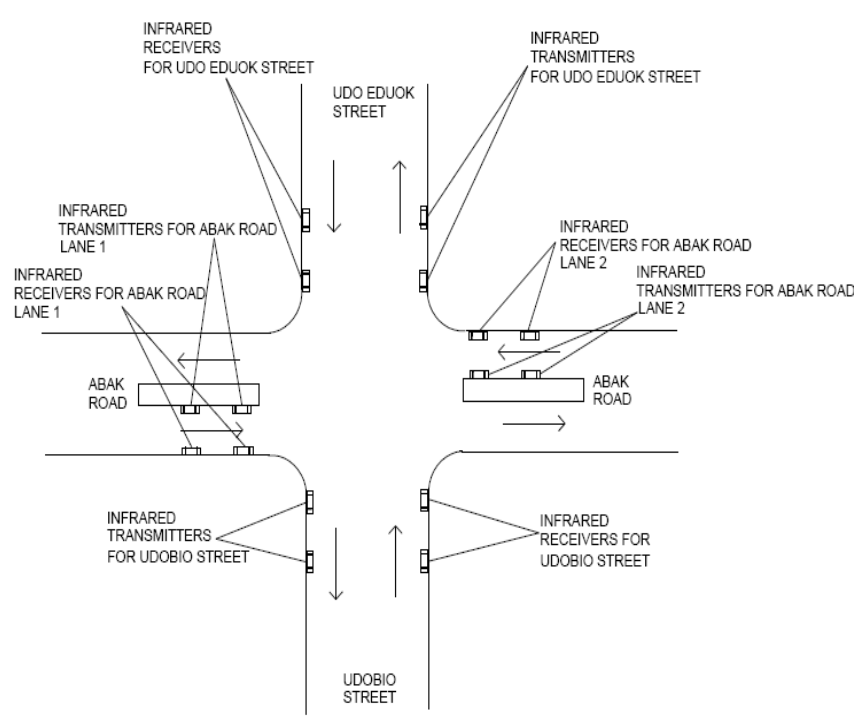

Figure 6: Infrared transmitter and receiver positions at location of implementation in Uyo Metropolis.

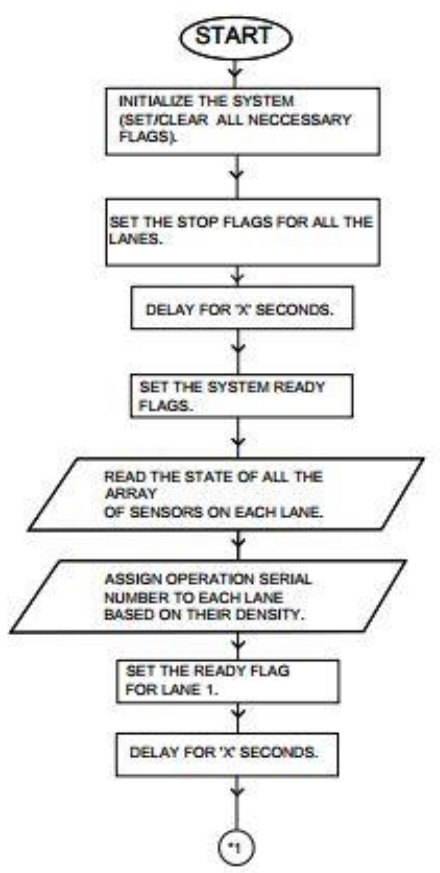

Figure 7(a): Flow chart of system. 


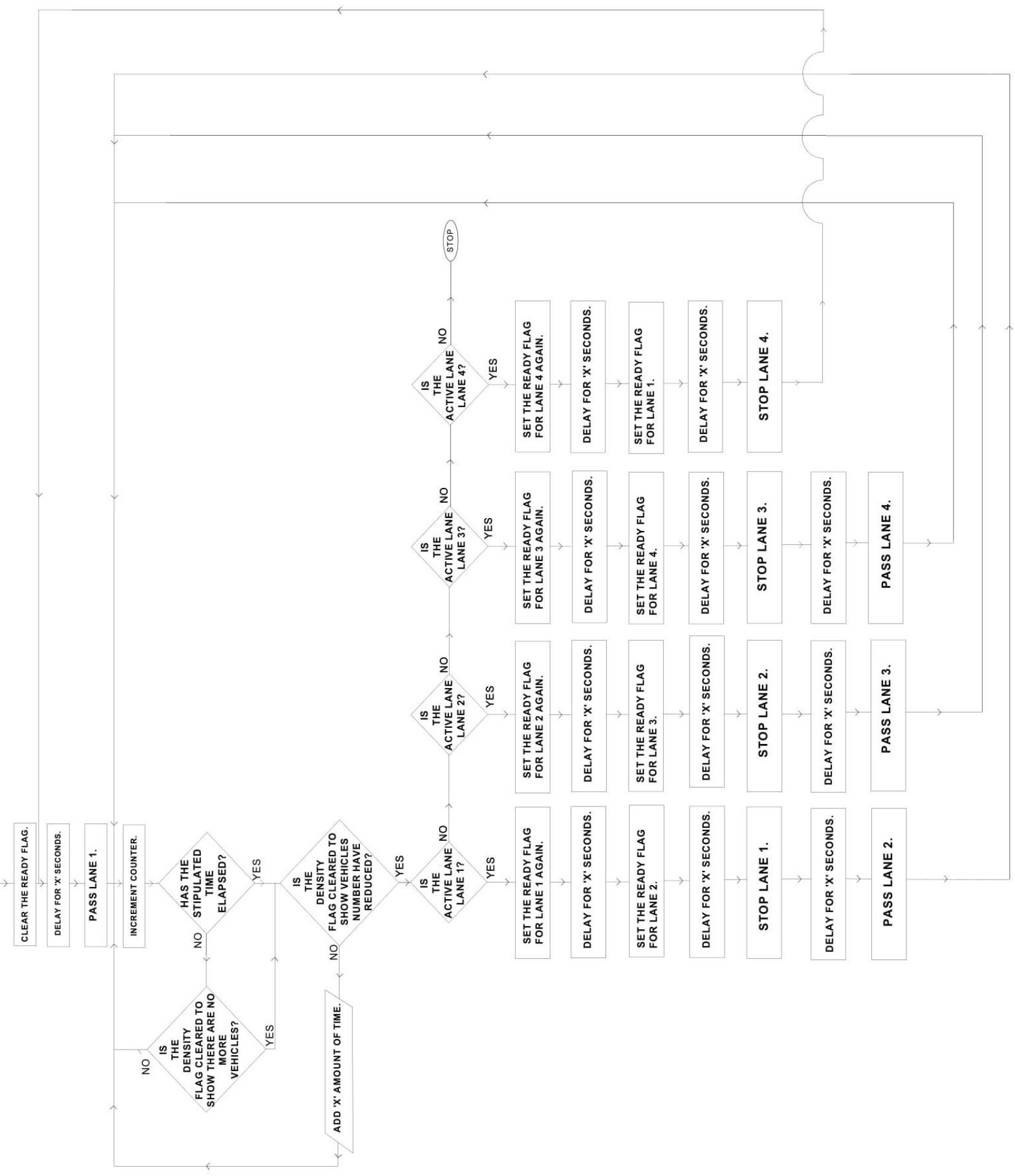

Figure 7(b): Flow chart of system.

\subsection{ALGORITHM}

The algorithm of the entire system is represented in the flow chart. The flow chart begins in Figure 7(a) and continues in Figure 7(b) for the purpose of legibility.

At the beginning of the flow chart, the system starts and so does the RAM of the microcontroller. It clears and initializes all the flags of the microcontroller. Initially, all the stop flags are set which consequently means that all the traffic light indicators for traffic control for all the four lanes show a RED light which stops all traffic at the beginning of its operation. The states of all the sensor arrays on each lane of traffic is then read and given as input to the microcontroller for logical operations, the system then goes further to assign operation serial number to each lane based on their densities and the lane with the most density is 
assigned lane one. Therefore, the algorithm is established and represented by a flowchart in Figure 7(a) and Figure 7(b). The flowchart is then translated into $\mathrm{C}$ language and compiled using the Microsoft Visual Studio IDE.

\subsubsection{Design Simulation}

The simulation of the circuit was performed using the Proteus software. Due to the absence of an infrared sensor representation in the Proteus software, switches were used to represent the sensors on each lane of traffic. A screenshot of the simulation procedure for the circuit is shown in Figure 8. The switches represent the infrared sensor [15] on each lane of traffic and the LEDs which represent the traffic indicator of each lane are divided into four and are controlled by the pins of the microcontroller. The microcontroller ports that were mainly used to control the traffic light indicators are PORT C and PORT D.

For PORT C, the pins used are RC0, RC1, RC2, RC6 and RC7 which control LEDs R1, Y1, G1, Y3, and G3 respectively while for PORT $\mathrm{D}$, the pins used are RD0RD7 for controlling LEDs R2, Y2, G2, R3, R4, Y4, and G4 respectively. Accordingly, each lane of traffic has three sets of LED bulbs for traffic control on each lane. Lane one has R1, Y1 and G1; lane two has R2, Y2 and G2, lane three has R3, Y3, and G3, lane four has R4, Y4 and G4 respectively; each colour of the LED having their usual meaning in traffic control.

The switches that are in Figure 8 represent the infrared sensors since there are no infrared representations in Proteus for simulation. The switches which are labeled; A11, A12, A21, A22 represent the infrared sensor array for lane one, switches; B11, B12, B21, B22 represent the infrared sensor array for lane two; switches C11, C12, C21, C22 represent the infrared sensors for lane three; switches D11, D12, D21, D22 represent the infrared sensors for lane four. For lane one, switches A11, A12, A21, A22 are interfaced with PORT A of the microcontroller with pins RA0-RA3 respectively; switches B11 and B12 for lane two are interfaced with PORT A pins RA4 and RA5 respectively and switches B21 and B22 are interface with PORT E, pins RE0 and RE1 respectively; for lane three, switches C11, C12, C21, C22 are interfaced with PORT B, pins RB0-RB3 respectively; for lane four, switches D11, D12, D21, D22 are interfaced with PORT B, pins RB4-RB7 respectively. Infrared sensors are active low at normal state which in this design portrays a scenario where there are no obstacles between the transmitter and the receiver, but if they are blocked, the receivers no longer receives pings from the transmitter and are switched to the active high state. This represents the function the switches perform; when the switches are open, they are in the active low state, but when they are closed, they are in the active high state.

To mimic the real life scenario, switch A22 is the last sensor, switch A21 is the second to the last sensor, switch A12 is the second sensor and switch A11 is the first sensor. When a car enters lane one, it blocks the transmission to the receiver which is the sensor A22, moving further it blocks sensor A21, A12 and then A11. This swift process can be mimicked by closing switches A22, A21, A12 and A11. This action switches the sensors from active low to active high. This process also applies for the other sensors in the other lanes of traffic when a vehicle enters each lane respectively. Each of the switches is grounded respectively.

\subsubsection{Complete Circuit Diagram}

For the four lanes of traffic to be controlled, requirements according to the aim as described in this paper would demand more than 35 Input-Output pins. For the aim to be achieved with the available pins of the controller, the inputs from the four sensor arrays were multiplexed using AND gates as shown in Figure 9 which is the complete PCB circuit layout as designed using the Diptrace Schematic Capture Software; PORT U8 represents the sensor input port for lane 1; and so does PORT U9, U11, U12 represent the sensor input port for lane 2, lane 3 and lane 4 respectively. Additionally; PORT U10, U3, U4 and U5 represent the output ports for the traffic lights of lane 1, lane 2, lane 3 and lane 4 respectively. AND gates are used to reduce the complexity of the circuit. The surveillance camera is interfaced with the microcontroller using PORT U14 which is the camera module, a serial communication module as designed using the Diptrace software because there is no representation of this in the Proteus Software; where the connector for the camera is placed and is operated in the 8-bit mode.

\subsubsection{Mode of Operation}

Once the traffic control commences operation, the states of all the sensor arrays on each lane of traffic is read and given as input to the microcontroller for logical operations. The system assigns serial number to each lane based on their density, where the lane with the most density is assigned lane one. Accordingly, the system sets the ready flag for lane one where the YELLOW light shows; in preparation for the passing of traffic in that lane and delays for a certain time before giving the go signal with the GREEN light. 


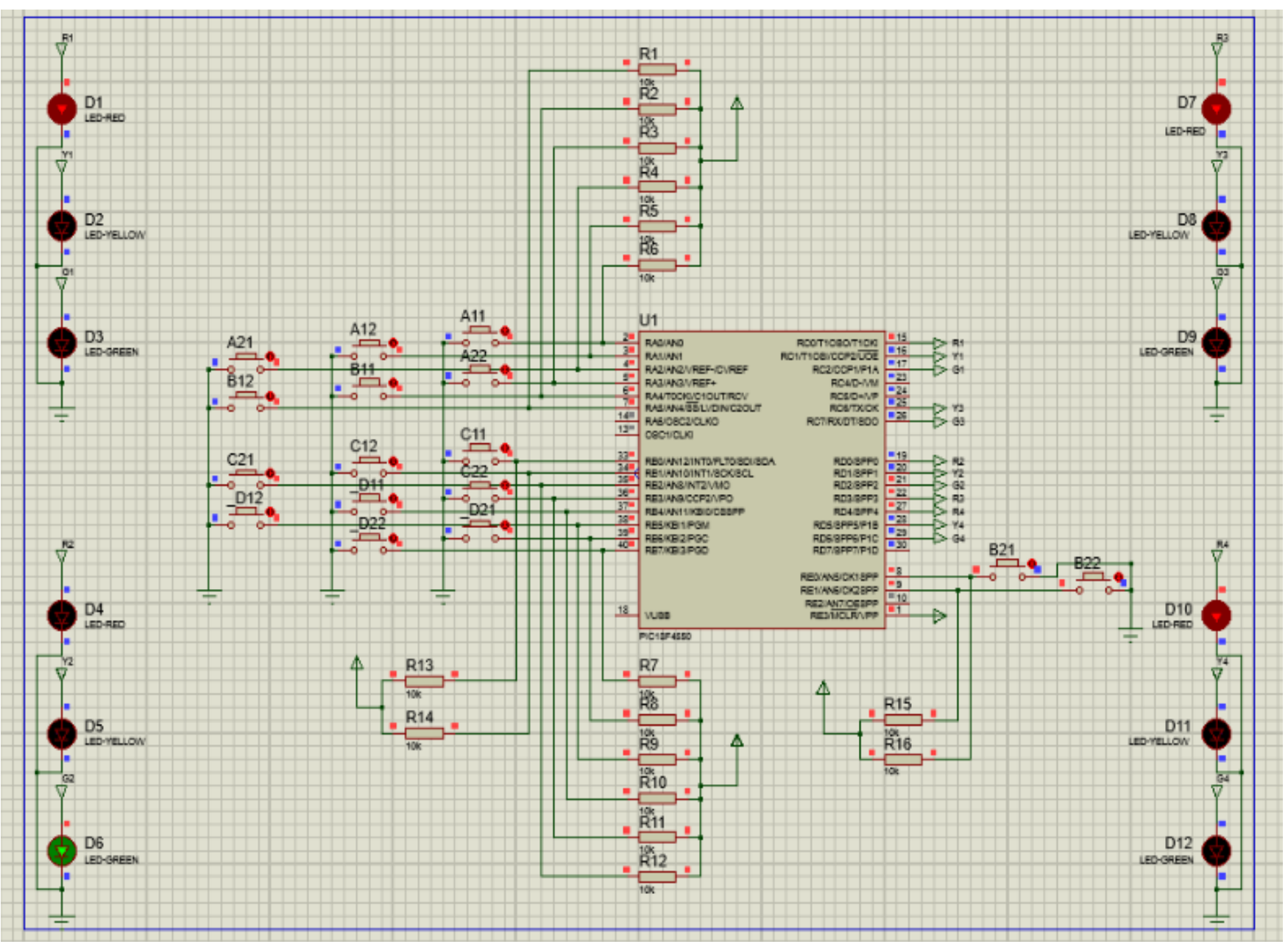

Figure 8: Software Simulation on Proteus 7.

Going further, to implement the primary loop of the flow chart; the system increments the counter where the amount of time allotted for lane one is accounted for; and takes a decision if the go time for lane one has elapsed. If it has not, the system takes a decision on the density of traffic on lane one by checking if the density flag has been cleared; If no, the system increments the counter again. After the density flag has been cleared and time has elapsed, the system makes a decision further to verify that the density flag has been cleared, if not, additional time is added and the primary loop is performed again. If the density flag for lane one is cleared, it makes a decision by checking to see if either lane one or any of the other three lanes are active, if not, the program stops.

If lane one is the active lane, the ready flag for that lane is set again and the YELLOW light shows in preparation to stop traffic on that lane while simultaneously setting the ready flag for lane two. Once lane one has been stopped, the YELLOW light at lane two shows indicating readiness and eventually the lane is passed and the primary loop of the system could be performed again. This process is repeated for all the other lanes depending on which one is the active lane.

For the surveillance system, a $360^{\circ}$ dome camera is used and mounted on top of each traffic light. When the GREEN light shows for a particular lane of traffic at any instant of time, the camera automatically rotates and faces the oncoming traffic but when the RED light shows, the camera rotates and faces the opposite direction that the vehicles are headed; such that if a car beats the traffic light of the associated lane, the surveillance camera captures the license number plate and stores it in a memory. If a road user beats traffic multiple times, the controller compares the captured number plate with that which exists already in the memory; it discards the captured image. The captured image is sent to the memory in streams of 8-bit binary code to match its 8-bit operating mode. The image stored in the memory (RAM or EPROM) can be copied to a removable storage device and given to law enforcement agencies. All these operations are performed by the logic operations carried out by the microcontroller through series of codes embedded in the controller. 


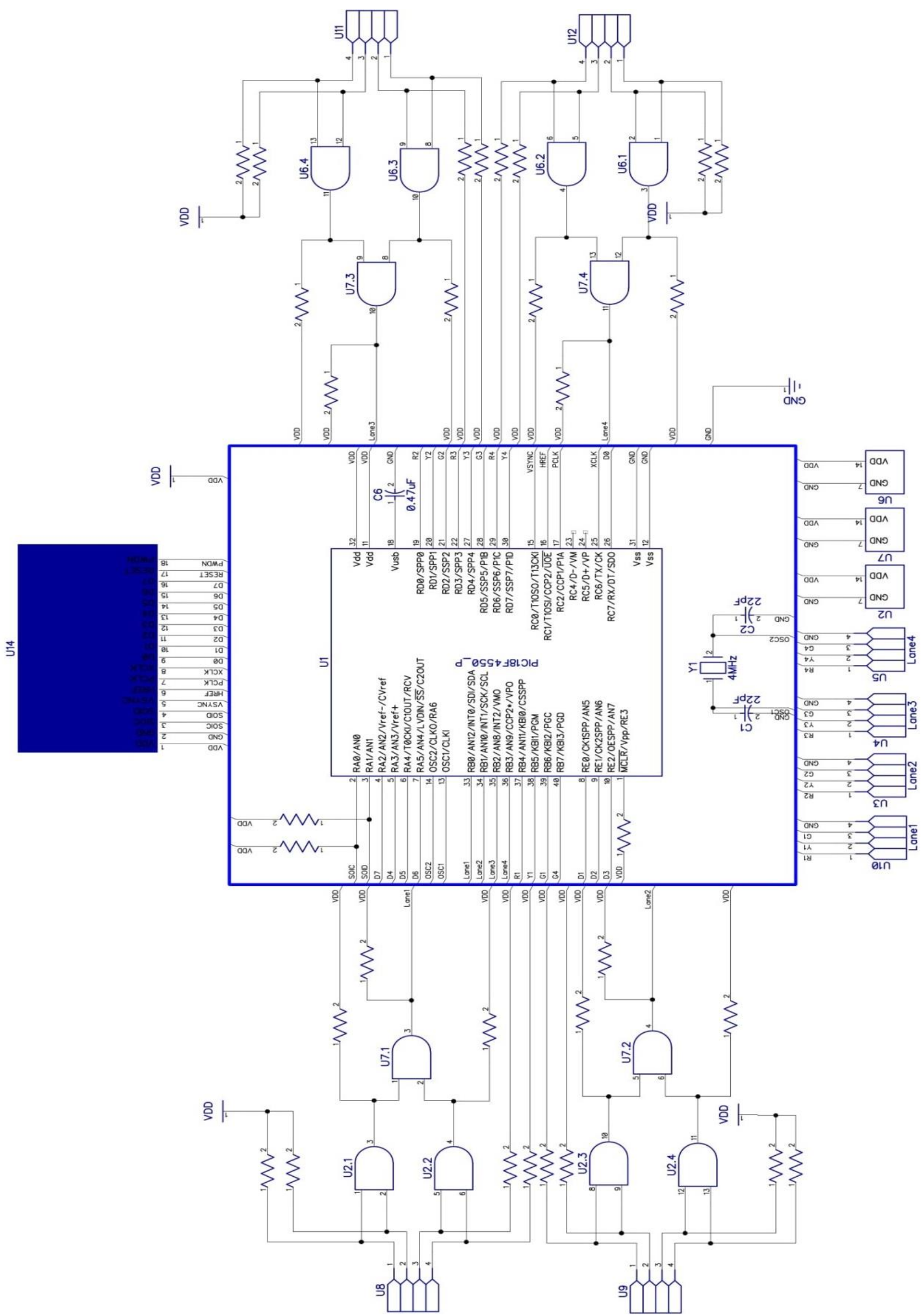

Figure 9: Complete Circuit Diagram. 

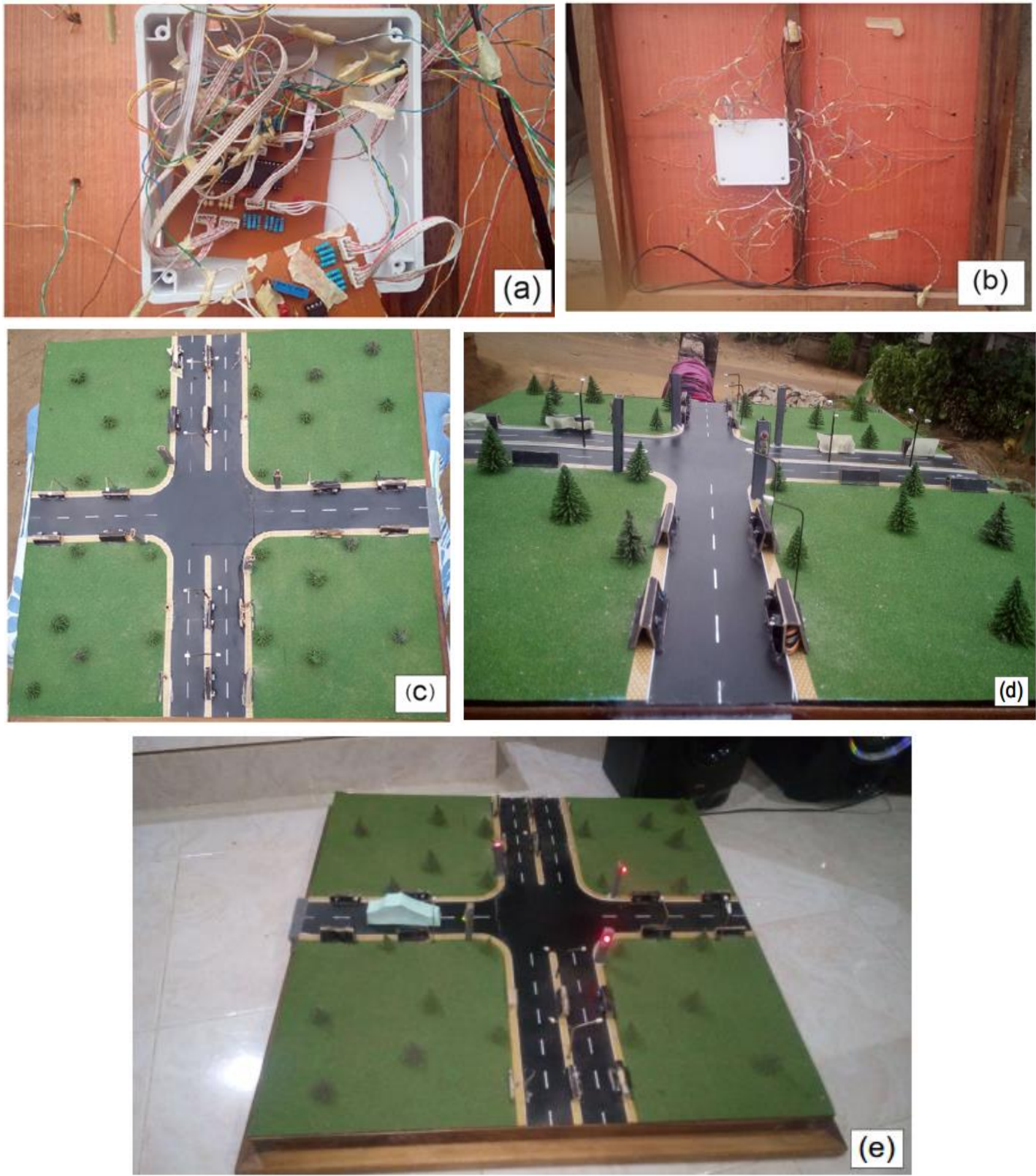

Figure 10: Stages of prototype implementation: (a) Microcontroller circuit mounted and soldered on Vero Board. (b) Control unit of system enclosed in box. (c) Density based traffic light control system. (d) Density based traffic light control system view through one of the lanes. (e) Density based traffic light control system in operation.

\section{CONCLUSION}

In this design work, a density based traffic light control system was developed for traffic control at ' + ' road intersection to reduce unnecessary time wastage and minimize road traffic casualties which the existing conventional traffic light control system has failed to achieve. As demonstrated by the test results in the simulation and the prototype implementation as shown in Figure 10 (a-e), the design has shown that the system developed is a viable tool for traffic control and the incorporation of a surveillance system would help reduce road casualties caused by road users who 
ignore traffic signals. Lastly, the objectives of the design were achieved. This paper has presented a means of controlling traffic at ' + ' road intersection using infrared sensors with an embedded microcontroller chip. Specifically, it demonstrates a working software solution for controlling traffic based on the density of traffic on each lane at the intersection. It provides a means of succor away from the conventional traffic light associated with even timing of lanes of traffic irrespective of the number of vehicles on the lanes per kilometer which is the density associated with that lane. This project as resourceful as it has proven to be can be improved upon by; the incorporation of renewable energy sources for 24 hours performance of the system. The system could also be designed to transmit captured vehicle plate numbers of defaulters in real time to relevant traffic agencies. Lastly, the design can be modified to control more than four lanes of traffic.

\section{ACKNOWLEDGEMENT}

The authors would like to thank Mr. Iyakkensemse Okon for his immense contributions.

\section{REFERENCES}

[1] Day, L. and McNeil, I. Biographical dictionary of the history of technology, Taylor and Francis, 1996.

[2] Moyer, S. "Mr. Trafficlight". Motor News. Automobile Club of Michigan: pp.14-15, 1947.

[3] Angus, P. D. 'Modeling of Traffic Signal Control and Transit Signal Priority'. Massachusetts Institute of Technology, 2001.

[4] Ashish, J., Manisha, M., Harish, V. and Amrita R. "Traffic Density Measurement based On-road Traffic Control using Ultrasonic Sensors and GSM Technology", in proc. AEEE $4^{\text {th }}$ International Conference on Emerging Trends in Engineering and Technology, Kurushetra, Haryana, India, October 25-27, 2013, pp. 778-786.

[5] Hashim, N. M., Jaafar, A. S., Ali, N.A., Salahuddin, L., Mohamad, N. R. and Ibrahim, M. A. "Traffic Light Control System for Emergency Vehicles Using Radio Frequency", ISOR Journal of Engineering, 3(7), pp. 43-52, 2013.
[6] Ganiyu, R. A., Arulogun, 0. T. and Okediran, 0. 0. "Development of a Microcontroller Based Traffic Light System for Road Intersection Control", International Journal of Scientific and Technology Research, 3(5), pp. 209-212, 2014.

[7] Kavya, P. W. and Joythi, S. "Traffic Light Control System Using Image Processing", International Journal of Innovative Research in Computer and Communication Engineering, 2(5), pp. 288-292, 2014.

[8] Mohit, D. S., Prema, Shubhendu, S., Sumedha, S. and Utkarsh, T. "Smart Traffic Control System Using PLC and SCADA", International Journal of Innovative Research in Science, Engineering and Technology, 1(2), pp. 169-172, 2012.

[9] Mani, T., Kumar, A. N., Dhanapal, M., Kumar, A. S., Hemalatha, R. and Vinodhini, I. "Density Based Traffic Light Control System". International Journal of Advanced Research in Management, Architecture, Technology and Engineering, 2(9), pp. 334-339, 2016.

[10] Microchip (2007). 28/40/44-Pin, High Performance; Enhanced Flash, USB Microcontrollers with nanoWatt Technology, PIC18F2455/2550/4455/4550 datasheet, Microchip Technology Inc., Arizona, USA.

[11] Mehta, V. K. and Rohit, M. Principles of Electronics. S. Chand and Company PVT. LTD., New Delhi, India, 1980.

[12] Malik, T. and Hongchi, S. "Adaptive Traffic Light Control with Wireless Control Networks", in $4^{\text {th }}$ IEEE Consumer Communications and Networking Conference, Las Vegas, Nevada, USA, January 1113, 2007, pp. 187-191.

[13] Khalil, Y., Al-Karaki, J. and Ali, S. “Intelligent Traffic Light Control System Using Wireless Sensors Networks", Journal of Information Science, Engineering and Technology, 26, pp. 753-768, 2010.

[14] Osigwe, U. C., Oladipo, O. F. and Onibere, E. A. "Design and Simulation of an Intelligent Traffic Control System", International Journal of Advances in Engineering and Technology, 1(5), pp. 47-57, 2011.

[15] Sharma, E. P., Mishra, A. and Singh, K. "Density Based Intelligent Traffic Control System Using IR Sensors", International Journal of Scientific Research, 4(5), pp. 3-4, 2015. 